\title{
EASL-EASD-EASO clinical practice guidelines for the management of non-alcoholic fatty liver disease in severely obese people: do they lead to over-referral?
}

\author{
Emilie Blond $^{1} \cdot$ Emmanuel Disse $^{2,3} \cdot$ Charlotte Cuerq $^{1} \cdot$ Jocelyne Drai $^{1}$. \\ Pierre-Jean Valette $^{4} \cdot$ Martine Laville $^{2,3} \cdot$ Charles Thivolet $^{2,3} \cdot$ Chantal Simon $^{2,3}$. \\ Cyrielle Caussy ${ }^{2,3}$
}

Received: 21 December 2016/Accepted: 6 March 2017/Published online: 28 March 2017

(C) Springer-Verlag Berlin Heidelberg 2017

\begin{abstract}
Aims/hypothesis We aimed to assess the application of the recent European Association for the Study of the Liver (EASL)-European Association for the Study of Diabetes (EASD)-European Association for the Study of Obesity (EASO) clinical practice guidelines for the management of non-alcoholic fatty liver disease (NAFLD) in severely obese individuals in routine clinical practice.

Methods We performed a single-centre retrospective observational study of 385 patients referred for severe obesity $\left(\mathrm{BMI} \geq 35 \mathrm{~kg} / \mathrm{m}^{2}\right.$ ) to our Endocrinology, Diabetes and Nutrition department, between 1 November 2014 and 31 December 2015. The recent EASL-EASD-EASO clinical practice guidelines for the management of NAFLD were retrospectively applied to the cohort using, successively, the NAFLD fibrosis score (NFS) and a combination of the NFS
\end{abstract}

Electronic supplementary material The online version of this article (doi:10.1007/s00125-017-4264-9) contains peer-reviewed but unedited supplementary material, which is available to authorised users.

Cyrielle Caussy

cyrielle.caussy@chu-lyon.fr

1 Biochemistry Department, Lyon Sud Hospital, Hospices Civils de Lyon, Claude-Bernard Lyon 1 University, Pierre Bénite, France

2 Department of Endocrinologie, Diabète, Nutrition, Centre Intégré de l'Obésité Rhône-Alpes, Fédération Hospitalo-Universitaire DO-iT, Lyon Sud Hospital, Hospices Civils de Lyon, Claude-Bernard Lyon 1 University, 165 Chemin du Grand Revoyet, 69495 Pierre-Bénite Cedex, France

3 Laboratoire CarMeN, Unité Inserm U1060 - INRA 1235 INSA-Lyon, Pierre Bénite, France

4 Department of Radiology, E. Herriot University Hospital, Hospices Civils de Lyon, Claude-Bernard Lyon 1 University, Lyon, France and transient elastography (TE) measurement in a subgroup of individuals.

Results We identified 313 (81.3\%) individuals with NAFLD in the cohort. The application of the EASL-EASD-EASO guidelines using NFS would lead to referral to a specialist for up to 289 individuals $(75.1 \%)$ in the cohort. The combination of NFS and TE measurement reclassified $28(25 \%)$ individuals from the medium/high risk group to low risk and would lead to the referral of $261(67.7 \%)$ individuals to a specialist. These proportions appear to be excessive given the expected prevalence of advanced fibrosis and nonalcoholic steatohepatitis (NASH) of around $10 \%$ and $30 \%$, respectively, in the severely obese population.

Conclusions/interpretation This is the first study to assess the strategy proposed by the EASL-EASD-EASO clinical practice guidelines for the management of NAFLD in severely obese individuals. The retrospective application of the guidelines in a cohort representing the routine clinical practice in our department would lead to an excessive number of specialist referrals and would also lead to an unjustified increase in health costs. Biomarkers and specific strategy for the screening of NASH and advanced fibrosis in morbidly obese individuals are thus crucially needed and would help to improve the actual guidelines.

Keywords Fibrosis $\cdot$ NAFLD fibrosis score $\cdot$ Non-alcoholic fatty liver disease $\cdot$ Non-alcoholic steatohepatitis · Obesity . Transient elastography
Abbreviations
ALT Alanine transaminase
AST Aspartate aminotransferase
EASL European Association for the Study of the Liver 


$\begin{array}{ll}\text { EASO } & \text { European Association for the Study of Obesity } \\ \text { GGT } & \gamma \text {-Glutamyltransferase } \\ \text { NAFLD } & \text { Non-alcoholic fatty liver disease } \\ \text { NASH } & \text { Non-alcoholic steatohepatitis } \\ \text { NFS } & \text { NAFLD fibrosis score } \\ \text { SWE } & \text { Shear wave elastography } \\ \text { TE } & \text { Transient elastography }\end{array}$

\section{Introduction}

Non-alcoholic fatty liver disease (NAFLD) is a major cause of chronic liver disease worldwide, especially in western countries [1]. Its prevalence is predicted to rise along with increasing rates of obesity, type 2 diabetes and metabolic syndrome [2]. Moreover, type 2 diabetes and obesity are independent risk factors for the progression of NAFLD to non-alcoholic steatohepatitis (NASH) and advanced fibrosis which increase the risk of progression to cirrhosis, liver-related mortality and hepatocellular carcinoma $[2,3]$ as well as increasing cardiovascular and overall mortality $[4,5]$. The recent European Association for the Study of the Liver (EASL)-European Association for the Study of Diabetes (EASD)-European Association for the Study of Obesity (EASO) guidelines recommend a systematic screening for NAFLD in individuals with a high metabolic risk and suggest the use of noninvasive biomarkers, such as NAFLD fibrosis score (NFS) potentially completed with transient elastography (TE) assessment, to identify individuals suspected to have advanced fibrosis for specialist referral [6].

We aimed to assess the application of the recent EASLEASD-EASO guidelines to severely obese individuals in routine clinical practice.

\section{Methods}

Population and study design This is a single-centre retrospective observational study of the individuals referred for severe obesity (BMI $\geq 35 \mathrm{~kg} / \mathrm{m}^{2}$ ) to the department of Endocrinology, Diabetes and Nutrition at Lyon South Hospital, Pierre-Bénite, France, between 1 November 2014 and 31 December 2015. The data were collected retrospectively using a specifically dedicated computerised database. We identified 385 individuals referred for severe obesity and retrospectively applied the recent European guidelines to this cohort. All the data processed were collected in the context of usual care. In line with the current French law, the secondary use of these data for scientific purposes does not require a preliminary ethical review. In addition, all data have been anonymised prior to re-use for scientific purposes and therefore, preliminary specific information to the individual is not required under French law.
Measurements Anthropometric data were collected for each individual. Biological data were measured and automatically extracted using computerised laboratory software (GLIMS V9, MIPS, Belgium) from the department of Biology in Lyon South Hospital (Pierre-Bénite, France). Blood samples were collected in the morning after an overnight fast for measurement of glucose (hexokinase assay), $\mathrm{HbA}_{1 \mathrm{c}}$ (HPLC), liver enzymes (enzymatic assessment) and albumin (immunoturbidimetric assay). The assays used for biological measurement are detailed in the electronic supplementary material (ESM) Table 1. LDL-cholesterol was calculated using the Friedewald formula: LDL-cholesterol $=$ Total cholesterol - HDL-cholesterol - (triacylglycerols/2.2) in $\mathrm{mmol} / \mathrm{l}$. Platelet count was determined on the most recent Sysmex XN-9000 (Sysmex, Roissy CDG, France), which is a fully automated haematology system that includes analytical modules with specific detection channels. For the determination of the platelet variable, we used the PLT-T channel, which allows the platelet count by fluorescence, after partial lysis of the platelet membrane by a reagent (Cellpack-DFL, Sysmex, Roissy CDG, France) and a labelling of the amino acids by a fluorochrome (Fluorocell PLT, Sysmex, Roissy CDG, France).

Abnormal liver enzymes were defined using the laboratory threshold: aspartate aminotransferase (AST) $>34 \mathrm{U} / \mathrm{l}$, alanine transaminase (ALT) $>55 \mathrm{U} / \mathrm{l}$. The upper normal thresholds for $\gamma$-glutamyltransferase (GGT) in men and women were $>64 \mathrm{U} / 1$ and $>36 \mathrm{U} / 1$, respectively. Glucose intolerance was determined by either fasting glycaemia $\geq 6 \mathrm{mmol} / 1, \mathrm{HbA}_{1 \mathrm{c}} \geq 6 \%$ $(42.1 \mathrm{mmol} / \mathrm{mol})$ or glycaemia $120 \mathrm{~min}$ after $75 \mathrm{~g}$ OGTT $\geq 8 \mathrm{mmol} / \mathrm{l}$. NFS was calculated using the formula in reference [7].

Abdominal ultrasound echography data were extracted from the radiology department server from Lyon University Hospital (GE Medical Systems 2006, Centricity, Enterprise Web, GE Healthcare, Vélizy-Villacoublay, France). All individuals were fasted overnight before imaging, as per the departmental procedure for abdominal echography. Steatosis was considered where there was bright or hyperechoic liver relative to adjacent right kidney or spleen. A subgroup of individuals $(n=209)$ underwent liver TE using ShearWave technology (Aixplorer; Supersonic Imagine, Aix-enProvence, France). All TE using shear wave elastography (SWE) measurements were obtained by scanning the right liver lobe through the intercostal spaces during a 4-6 s breath hold. The elastography map in 2D-SWE was placed 1-2 cm below the liver capsule, at $3-5 \mathrm{~cm}$ from the transducer, avoiding vessels. Successful acquisition was considered only if more than two-thirds of the elastography map was homogeneously colour-coded with gradual colour transitions. Five to ten acquisitions were performed. Average (AVG) value and SD were then calculated, and only liver stiffness measurements with $(\mathrm{CV}=\mathrm{SD} / \mathrm{AVG})<0.3$ were considered to be 
reliable. The following elastography thresholds were used, in accordance with the manufacturer's manual: F0-F1, $6.4 \mathrm{kPa}$ (5.3-7.1); $\mathrm{F}>2,7.5 \mathrm{kPa}(7.0-8.5) ; \mathrm{F}<3,10.1 \mathrm{kPa}(9.5-11.6)$; $\mathrm{F}>4,14.4 \mathrm{kPa}(13.1-18.8)$.

Primary outcome The primary outcome was the assessment of the number of individuals screened for medium or high risk of advanced fibrosis or NASH, following the EASL-EASDEASO guidelines.

Statistical analysis Descriptive analyses were performed using SPSS 20.0 (IBM, SPSS, Chicago, IL, USA). Continuous variables are expressed as mean $\pm \mathrm{SD}$ and categorical variables as a percentage.

\section{Results}

Population characteristics Characteristics of the study population are presented in Table 1. The mean BMI was $43.2 \pm 6.4 \mathrm{~kg} / \mathrm{m}^{2}$, participants were mainly women $(69.6 \%)$ aged $47.3 \pm 14$ years. A total of $54.8 \%$ had a disorder of glucose homeostasis and $36.1 \%$ had type 2 diabetes. Abdominal ultrasound echography revealed that 313 $(81.3 \%)$ individuals exhibited NAFLD.

\section{Retrospective application of EASL-EASD-EASO guide-} lines to the cohort We retrospectively applied the EASLEASD-EASO guidelines for the screening of advanced fibrosis using the NFS (Fig. 1a). This strategy led to a referral to a specialist for $289(75.1 \%)$ individuals in the cohort. As the guidelines suggest that the combination of fibrosis score and TE could increase the diagnostic accuracy, we also studied the TE measurement available for a subgroup of 209 individuals. Of these, the TE measurement was non-contributive for 81 $(38.8 \%)$ individuals; $100(47.8 \%)$ were classified as stage F0-F1 and a minority was classified as stage F3-F4 (13, $6.2 \%$ ) (Table 1). The combination of NFS and TE would lead to the reclassification of 28 (25\%) individuals from the medium/high risk of fibrosis group to the low risk of fibrosis group. However, $261(67.7 \%)$ individuals would still require specialist referral using this combined screening strategy (Fig. 1b).

\section{Discussion}

We assessed the application of the EASL-EASD-EASO guidelines in a routine clinical practice setting in a cohort of 385 individuals referred to our department for severe obesity care. We identified 313 (81.3\%) individuals with NAFLD in the cohort. The retrospective application of EASL-EASDEASO guidelines would have led to the referral to a specialist
Table 1 Characteristics of the study population

\begin{tabular}{|c|c|}
\hline Characteristic & Value \\
\hline \multicolumn{2}{|l|}{ Demographics } \\
\hline Female sex, $n(\%)$ & $268(69.6)$ \\
\hline Age, years & $47.3 \pm 13.9$ \\
\hline Weight, kg & $119.2 \pm 22.8$ \\
\hline Height, m & $1.66 \pm 0.1$ \\
\hline BMI, $\mathrm{kg} / \mathrm{m}^{2}$ & $43.2 \pm 6.4$ \\
\hline \multicolumn{2}{|l|}{ Clinical variables } \\
\hline Diabetes or glucose intolerance, $n(\%)$ & $211(54.8)$ \\
\hline Type 2 diabetes, $n(\%)$ & $139(36.1)$ \\
\hline \multicolumn{2}{|l|}{ Biological variables } \\
\hline $\mathrm{HbA}_{1 \mathrm{c}}, \%$ & $6.14 \pm 1.23$ \\
\hline $\mathrm{HbA}_{1 \mathrm{c}}, \mathrm{mmol} / \mathrm{mol}$ & $43.6 \pm 13.4$ \\
\hline Fasting glucose, mmol/1 & $6.0 \pm 2.2$ \\
\hline C-reactive protein, $\mathrm{nmol} / 1$ & $102.2 \pm 110.9$ \\
\hline Albumin, g/l & $41.1 \pm 3.5$ \\
\hline Triacylglycerol, mmol/1 & $1.76 \pm 0.94$ \\
\hline Total cholesterol, mmol/1 & $5.17 \pm 1.21$ \\
\hline HDL-cholesterol, mmol/1 & $1.18 \pm 0.31$ \\
\hline LDL-cholesterol, mmol/1 & $3.21 \pm 1.06$ \\
\hline AST, U/l & $33.2 \pm 17.5$ \\
\hline Abnormal AST, $n(\%)$ & $109(28.3)$ \\
\hline ALT, U/1 & $37.5 \pm 25.6$ \\
\hline Abnormal ALT, $n(\%)$ & $62(16.1)$ \\
\hline GGT, U/1 & $52.0 \pm 71.9$ \\
\hline Abnormal GGT, $n(\%)$ & $140(36.4)$ \\
\hline AST:ALT ratio & $1.02 \pm 0.37$ \\
\hline Abnormal liver enzymes, $n(\%)^{\mathrm{a}}$ & $176(45.7)$ \\
\hline Platelets, g/1 & $282.6 \pm 77.7$ \\
\hline \multicolumn{2}{|l|}{ NAFLD assessment } \\
\hline NAFLD (US echography), $n(\%)$ & $313(81.3)$ \\
\hline \multicolumn{2}{|l|}{$\mathrm{NFS}, n(\%)^{\mathrm{b}}$} \\
\hline NFS 1 & $119(30.9) /$ \\
\hline NFS 2 & $189(49.1)$ \\
\hline NFS 3 & $77(20.0)$ \\
\hline \multicolumn{2}{|l|}{ TE, $n(\%)^{\mathrm{c}}$} \\
\hline F0-F1 & $100(47.8)$ \\
\hline $\mathrm{F} 2$ & $15(7.2)$ \\
\hline F3-F4 & $13(6.2)$ \\
\hline $\mathrm{NC}$ & $81(38.8)$ \\
\hline
\end{tabular}

Values are means $\pm \mathrm{SD}$, unless otherwise noted as $n(\%)$

${ }^{a}$ Any increase in AST, ALT or GGT

${ }^{\mathrm{b}}$ NFS thresholds were as follows: stage $1, \leq-1.455$ (predictor of absence of significant fibrosis, F0-F2 fibrosis); stage $2,>-1.455$ to $\leq 0.675$ (indeterminate score); stage $3,>0.675$ (predictor of presence of significant fibrosis, F3-F4 fibrosis)

${ }^{\mathrm{c}}$ TE measurements were classified by predictive grade of fibrosis according to METAVIR classification. TE measurements were available for $n=209$ individuals

NC, non-contributive; US, ultrasound 


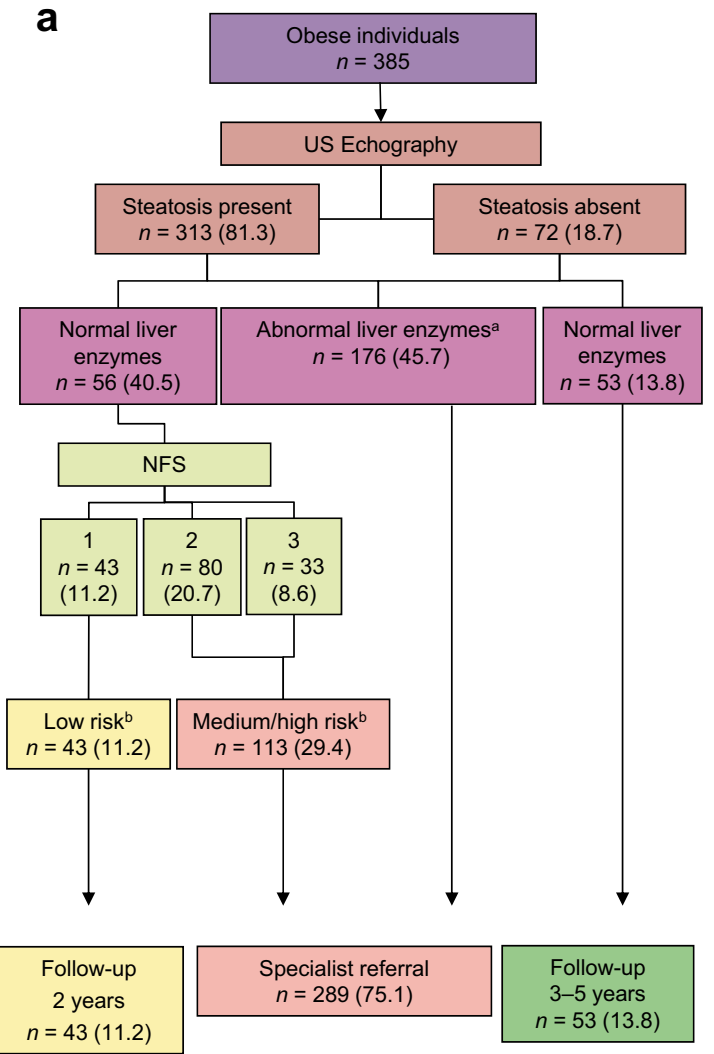

Fig. 1 Retrospective application of EASL/EASD/EASO guidelines to the cohort [6]. Flow chart using NFS to screen for advanced fibrosis (a) or using a combination of NFS and TE measurement: first the application of NFS and then consideration of the TE measurement in the subgroup classified with medium and high risk of fibrosis with NFS ( $n=113)$, TE measurement was available for 51 individuals in this subgroup (b). The results are presented as number of individuals, with percentage of the total cohort in parentheses. The thresholds for NFS used were as follows: stage

for 289 individuals $(75.1 \%)$ in the cohort. This appears to be excessive given the expected prevalence of advanced fibrosis and NASH of $10 \%$ and $30 \%$, respectively, in the severely obese population.

This study has limitations due to its retrospective and single-centre design. Details of treatment and alcohol uptake, which are potential confounders for NAFLD, were not available. The ultrasound echography and TE assessments were performed by several operators, potentially providing interpretation bias. The studied population was formed mainly of women due to selection bias, as women are more likely to consult for obesity care. Nevertheless, this cohort is a representative sample of the individuals routinely addressed to our department for severe obesity over the course of a year, and therefore enables assessment of the guidelines in routine clinical practice. Finally, the lack of liver biopsy did not allow accurate assessment of the efficiency of the guidelines.

The EASL-EASD-EASO guidelines recommend the systematic specialist referral of individuals with any abnormal liver enzymes, including ALT, AST and GGT. In our study,

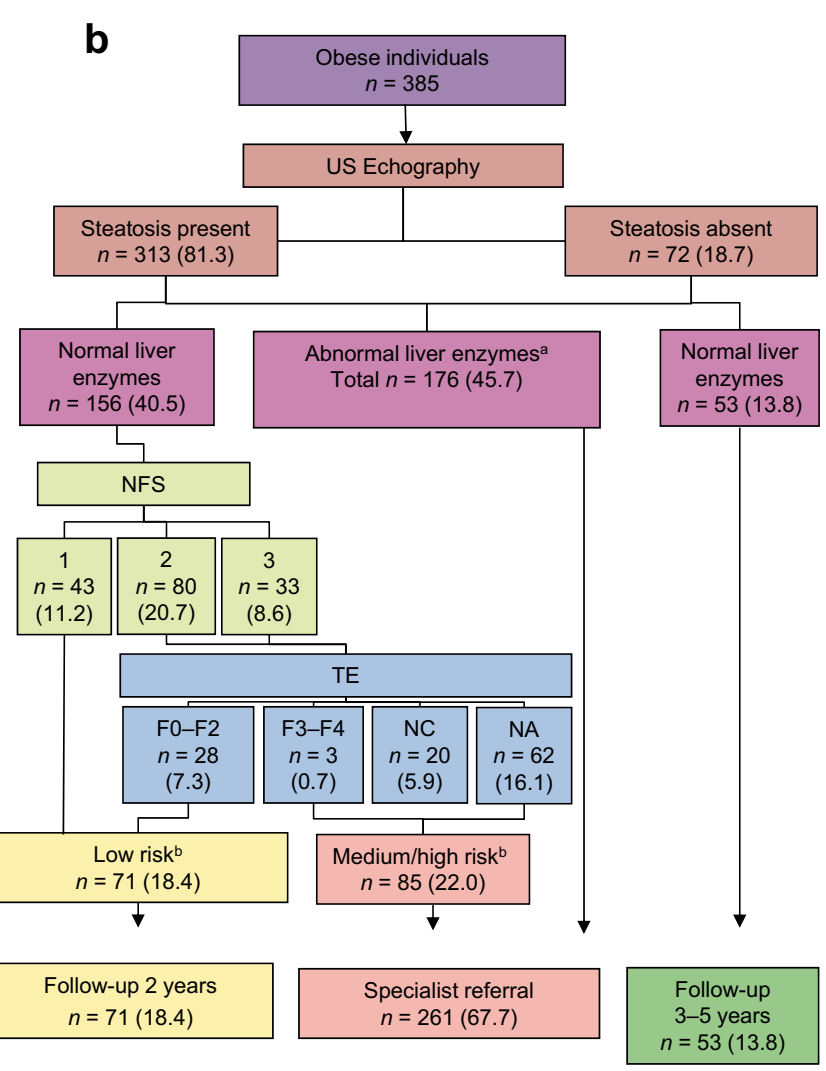

$1, \leq-1.455$ (predictor of absence of significant fibrosis, F0-F2 fibrosis); stage $2,>-1.455$ to $\leq 0.675$ (indeterminate score); stage $3,>0.675$ (predictor of presence of significant fibrosis, F3-F4 fibrosis). TE measurements are classified by predictive grade of fibrosis using the METAVIR classification. ${ }^{\mathrm{a}}$ Any increase in ALT, AST or GGT. ${ }^{\mathrm{b}}$ Low risk, indicative of no/mild fibrosis; medium/high risk, indicative of significant fibrosis or cirrhosis. NA, not available; NC, non-contributive; US, ultrasound

we were unable to capture important confounders of elevated liver enzymes, such as excessive alcohol intake or drugrelated or other liver diseases. Contrary to the practice guideline published by the American Gastroenterological Association, American Association for the Study of Liver Diseases and American College of Gastroenterology, the EASL-EASD-EASO guidelines do not recommend the screening of these factors in its proposed algorithm [8]. Thus, the strict application of the EASL-EASD-EASO algorithm leads to the identification of a high proportion of individuals with abnormal liver enzymes in our cohort ( $n=176,45.7 \%$ ), accounting for the high number of individuals recommended for specialist referral. A short follow-up period of measurement of liver enzymes after modification of potential factors associated with elevated liver enzymes or lifestyle intervention may be a more appropriate approach in the primary care setting. A reevaluation of an individual after 2-3 weeks may result in normal liver enzyme levels, with referral to specialist being required if this was not the case. 
The EASL-EASD-EASO guidelines recommend the use of any biomarkers of fibrosis. The NFS was chosen as it has been validated in several cohorts, including morbidly obese individuals $[9,10]$, and is easy to use in routine clinical care. The combination of NFS and TE in a subgroup of individuals in the cohort reclassified 28 (25\%) individuals in the medium/ high risk group to low risk but would still lead to the referral of $261(68 \%)$ individuals to a specialist. In addition, the rate of non-contributive TE measurement is important $(n=81$, $38.8 \%$ ), making this procedure less efficient in severely obese individuals. Therefore, this combined strategy needs to be validated in morbidly obese individuals.

This is the first study to assess the application of EASLEASD-EASO clinical practice guidelines for the management of NAFLD in severely obese individuals. The application of the guidelines to a cohort representing the population found in routine clinical practice would lead to the referral to a specialist of an excessive number of individuals and thus would lead to an unjustified increase in the health costs. Biomarkers and a specific strategy for the screening of NASH and advanced fibrosis in morbidly obese individuals are thus crucially needed and would help to improve the actual guidelines.

Acknowledgements We thank the staff of the department of Endocrinology and Nutrition, Radiology and Biology, Lyon Sud University Hospital for their helpful assistance.

Data availability The datasets are available from the corresponding author on reasonable request.

Funding This research received no specific grant from any funding agency in the public, commercial or not-for-profit sectors.

Duality of interest The authors declare that there is no duality of interest associated with this manuscript.

Contribution statement All authors made substantial contributions to the conception and design or acquisition, analysis and interpretation of data. All authors drafted the article or revised it critically for important intellectual content. All authors approved the final version of the manuscript to be published. $\mathrm{CCa}$ is the guarantor of this work.

\section{References}

1. Bellentani S (2017) The epidemiology of non-alcoholic fatty liver disease. Liver Int 37(Suppl 1):81-84

2. Younossi ZM, Koenig AB, Abdelatif D, Fazel Y, Henry L, Wymer M (2016) Global epidemiology of nonalcoholic fatty liver disease - meta-analytic assessment of prevalence, incidence, and outcomes. Hepatology 64:73-84

3. Dulai PS, Singh S, Patel J et al (2017) Increased risk of mortality by fibrosis stage in non-alcoholic fatty liver disease: systematic review and meta-analysis. Hepatology. doi:10.1002/hep.29085

4. Ekstedt M, Hagstrom H, Nasr P et al (2015) Fibrosis stage is the strongest predictor for disease-specific mortality in NAFLD after up to 33 years of follow-up. Hepatology 61:1547-1554

5. Angulo P, Kleiner DE, Dam-Larsen S et al (2015) Liver fibrosis, but no other histologic features, is associated with long-term outcomes of patients with nonalcoholic fatty liver disease. Gastroenterology 149:389-397 e310

6. European Association for the Study of the Liver, European Association for the Study of Diabetes, European Association for the Study of Obesity (2016) EASL-EASD-EASO clinical practice guidelines for the management of non-alcoholic fatty liver disease. Diabetologia 59:1121-1140

7. Angulo P, Hui JM, Marchesini G et al (2007) The NAFLD fibrosis score: a noninvasive system that identifies liver fibrosis in patients with NAFLD. Hepatology 45:846-854

8. Chalasani N, Younossi Z, Lavine JE et al (2012) The diagnosis and management of non-alcoholic fatty liver disease: practice guideline by the American Association for the Study of Liver Diseases, American College of Gastroenterology, and the American Gastroenterological Association. Hepatology 55:2005-2023

9. Ooi GJ, Burton PR, Doyle L et al (2017) Modified thresholds for fibrosis risk scores in nonalcoholic fatty liver disease are necessary in the obese. Obes Surg 21:115-125

10. Luger M, Kruschitz R, Kienbacher C et al (2016) Prevalence of liver fibrosis and its association with non-invasive fibrosis and metabolic markers in morbidly obese patients with vitamin D deficiency. Obes Surg 26:2425-2432 